der Pflegeversicherung - zu befürchten. In diesen Zeiten ist es umso wichtiger, das, was in den vergangenen 60 Jahren beim Abbau der Diskriminierung nach dem Geschlecht erreicht worden ist, zu verteidigen. Erreichte Erweiterungen gesetzlicher Leistungen sollen erhalten bleiben. Eine weitere Erosion der Sozialversicherungssysteme durch die Streichung der verbliebenen solidarisch umverteilenden Elemente gilt es zu verhindern. Dass Wettbewerb und Markt nicht der einzig gültige Maßstab sein kann, haben die letzten Monate deutlich gezeigt.

Zum anderen zeigt die Finanzmarktkrise deutlich, dass sich Armutsrisiken auch verwirklichen und Frauen hiervon ganz besonders getroffen sind: prekäre Beschäftigungsverhältnisse werden weiter zunehmen - betroffen sind zumeist Frauen. Unterbrochene Erwerbsbiografien führen zu niedrigen Rentenansprüchen - dies gilt weiterhin vor allem für Frauen. Hinzu kommt, dass das Ehegattensplitting und die Zusammenveranlagung von Eheleuten immer noch Frauen zur Aufgabe ihrer
Erwerbstätigkeit motivieren. Auch hieraus ergeben sich im Falle einer Scheidung Armutsrisiken. Berufsrückkehrerinnen werden es auf dem Arbeitsmarkt ohne entsprechende Qualifizierung noch schwerer haben. Die Anrechnung des Partnereinkommens beim SGB II führt zu einem Verlust von Leistungsansprüchen, der einen Wiedereinstieg in den Arbeitsmarkt weiter erschwert.

Ein Augenmerk sollte in näherer Zukunft daher insbesondere darauf liegen, wie durch strukturelle Veränderungen in Zukunft Frauen eigenständig und individuell abgesichert sein können, um Armut zu vermeiden und Teilhabe zu ermöglichen. Das djb-Rentenmodell ist ein Beispiel für einen solchen Vorschlag für eine strukturelle Veränderung sozialer Sicherungssysteme. Hier gilt es weiterzudenken.

Gleichstellungspolitik ist kein Luxus und kein Thema für gute Zeiten. Das Gebot des Art. 3 Abs. 2 GG ist keines, das man sich leisten können muss. Es gilt immer.

\title{
Gender Pay Gap mit neuen Instrumenten überwinden
}

Prof. Dr. Sibylle Raasch

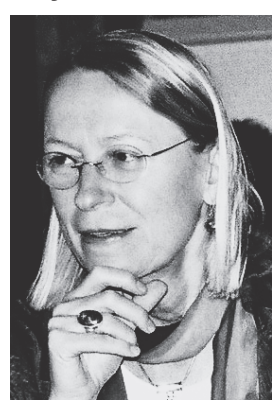

Mitglied der Kommission Arbeits-, Gleichstellungs- und Wirtschaftsrecht des djb; Hochschullehrerin, z. Zt. Universität Wien
Der Gender Pay Gap, also die Entgeltdifferenz zwischen Frauen und Männern, betrug im Jahr 2007 nach Angaben des Statistischen Bundesamtes ${ }^{1}$ in Deutschland 23 Prozent, sogar 24 Prozent in den alten Bundesländern, aber nur 6 Prozent in den neuen Bundesländern. Die Bundesrepublik ist in Sachen Entgeltgleichheit auch nicht auf einem „guten Weg“. Der Verdienstabstand wächst tendenziell weiter. Nur im Bereich Verkehr und Nachrichtenübermittlung hat sich der Abstand der durchschnittlichen Bruttostundenverdienste 2006 bis 2007 verringert - um gerade einmal ein Prozent. $^{2}$

Dieser Gender Pay Gap ist ein Ergebnis des Zusammenwirkens verschiedener Faktoren im Sinne struktureller Frauenbenachteiligung. Hierzu zählen insbesondere: die fortbestehende Abwertung von Tätigkeiten und Verhaltensweisen, die mit Frauen und Weiblichkeit identifiziert werden; die Dominanz des Familienernährer-Zuverdienerinnen-Modells als Lebensform; das Verbleiben der Haus- und Familienarbeit vorrangig bei den Frauen, selbst wenn partnerschaftlicher gelebt werden soll; die geschlechtsspezifisch getrennten Arbeitsmärkte in der Gesamtwirtschaft und in den einzelnen Betrieben, wobei die Frauen weniger Bereiche mit schlechterer Vergütung und schlechteren Aufstiegschancen besetzen; die geringe Vereinbarkeit von Beruf und Familie mit der Folge, dass Frauen ihre Erwerbstätigkeit häufiger und länger unterbrechen als Männer und deswegen erhebliche Gehaltseinbußen hinneh- men müssen ${ }^{3}$ oder in Teilzeitarbeit ausweichen; die Diskriminierung von Teilzeitarbeit; die Diskriminierung von Frauen bei der Einstellung und beim beruflichen Aufstieg; die unverändert extreme Unterrepräsentanz von Frauen in Führungs- und Leitungspositionen; verschiedene Formen von Entgeltdiskriminierung. ${ }^{4}$

Der Gender Pay Gap muss also durch Maßnahmen in allen diesen Bereichen bekämpft werden. Die bessere Vereinbarkeit von Beruf und Familie ist hierbei nur ein Mosaiksteinchen, nicht die Lösung des Gesamtproblems, wie es Erklärungen der Bundesregierung aber häufig nahelegen. Auf ein gezieltes Vorgehen gegen direkte und mittelbare Entgeltdiskriminierung kann aus Frauensicht keinesfalls verzichtet werden.

\section{Verbesserungen auf freiwilliger Basis nicht zu er- warten}

Die Vereinbarung zwischen der Bundesregierung und den Spitzenverbänden der deutschen Wirtschaft zur Förderung der Chancengleichheit von Frauen und Männern aus dem Jahr 2001 setzt auf

1 Vgl. Statistisches Bundesamt, PM Nr. 427 vom 14.11.2008.

2 Vgl. Statistisches Bundesamt a.a.O.

3 Vgl. neuste Berechnungen von Boll, Christina: Lohneinbußen durch geburtsbedingte Erwerbsunterbrechungen..., HWWI Research Paper 1-19, Hamburg Januar 2009.

4 Vgl. Droßard, Ralf: Verdienstabstand zwischen Frauen und Männern, Destasis, 26.8.2008. 
reine Freiwilligkeit. Sie hat in der Antidiskriminierungs- und Gleichstellungspolitik keinerlei Durchbruch gebracht. Freiwillig werden nur wenige und die immer gleichen Unternehmen gleichstellungspolitisch aktiv. Das zeigen die inzwischen drei Bilanzen Chancengleichheit zu dieser Vereinbarung, die sämtlich nicht mit verbesserten Zahlen und Entwicklungstendenzen, sondern nur mit positiven Einzelfällen argumentieren. ${ }^{5}$ Es bedarf eines verstärkten gesetzlichen Drucks hin zu mehr Chancengleichheit durch ein wirksameres Allgemeines Gleichbehandlungsgesetz (AGG) und ein Gleichstellungsgesetz für die Privatwirtschaft, das die Wirtschaft über bloße Nichtdiskriminierung hinaus auch zu positiven Gleichstellungsmaßnahmen zwingt. Nur auf ersteres, die notwendigen Verbesserungen des Diskriminierungsschutzes, soll im Folgenden näher eingegangen werden.

\section{Wenig Veränderungsdruck durch das AGG}

Die Unternehmen werden durch das neue AGG nicht unter besonderen Veränderungsdruck gesetzt, wie die weiterhin geringe Anzahl von Diskriminierungsklagen ${ }^{6}$ und eine Hamburger Unternehmensbefragung zu den durchgeführten Veränderungen ${ }^{7}$ zeigen. Die meisten Unternehmen haben nur ihre Stellenanzeigen um Hinweise auf mögliche Diskriminierungen entschärft, ihre bisherige Einstellungs-, Beförderungs- und Entgeltpolitik jedoch beibehalten.

\section{Verbot von Entgeltdiskriminierung nur unzulänglich im AGG geregelt}

Das AGG behandelt Entgeltgleichheit im Gegensatz zum vorausgegangenen $\mathbb{S} 612$ Abs. 3 BGB nicht einmal mehr ausdrücklich. Im Anwendungsbereich findet sich unter $\mathbb{} 2 \mathrm{Abs}$. 1 Nr. 2 AGG lediglich lakonisch die Formulierung „Arbeitsbedingungen einschließlich Arbeitsentgelt“. $\$ 8$ Abs. 2 AGG nennt Entgeltgleichheit nur noch im Zusammenhang mit Ausnahmen vom Gleichbehandlungsgrundsatz. Das Problem der Entgeltdiskriminierung wird durch das AGG also gesetzlich erneut unsichtbar gemacht. Die in Artikel 1 Abs. 1 der Richtlinie 75/117/EWG enthaltenen Bestimmungen und Präzisierungen zum Grundsatz des gleichen Entgelts bei gleicher Arbeit oder bei einer Arbeit, die als gleichwertig anerkannt wird, sind im deutschen Recht weder im AGG noch in einer anderen Bestimmung umgesetzt. Es fehlt auch ein ausdrücklicher Bezug auf den in Artikel 141 EG niedergelegten Anspruch auf gleiches Entgelt für gleiche oder gleichwertige Arbeit. Der Gesetzgeber sollte daher in das AGG ein ausdrückliches und differenziert geregeltes Verbot von Entgeltdiskriminierung aufnehmen, um dem Anspruch nach diskriminierungsfreier Entlohnung mehr Nachdruck durch Sichtbarkeit zu verleihen. Zudem kann gerade die Anwendung diskriminierender Tarifverträge wegen $\mathbb{} 15$ Abs. 3 AGG (Entschädigung nur bei grober Fahrlässigkeit des Arbeitgebers/der Arbeitgeberin) bislang kaum effektiv sanktioniert werden. Diese Privilegierung von Tarifverträgen, die in den EU-Antidiskriminierungsrichtlinien keinerlei Grundlage hat, ist $\mathrm{zu}$ streichen.
Jedoch würde das Allgemeine Gleichbehandlungsgesetz auch dann noch erhebliche Mängel in der Rechtsdurchsetzung aufweisen, wenn diese augenfälligsten Mängel beseitigt wären. Denn es setzt weiterhin darauf, dass die Diskriminierten selbst durch ihre Einzelklagen die notwendigen allgemeinen Strukturveränderungen in Wirtschaft und Gesellschaft bewirken. Das aber können die Diskriminierten als gesellschaftlich ja gerade besonders durchsetzungsschwache Gruppe nicht bewirken, dazu fehlen ihnen Rechtskenntnisse, finanzielle Mittel und Durchhaltevermögen. Zudem treffen Klagen gegen einzelne Diskriminierungen meist nur Teilaspekte einer viel umfassenderen Diskriminierungsstruktur und oft nicht einmal die wirkungsmächtigsten. Und die Bindungswirkung eines positiven Gerichtsentscheids erstreckt sich immer nur auf den eingeklagten Einzelfall.

Das gilt ganz besonders bei Klagen gegen Entgeltdiskriminierung. Denn hier geht es zumeist um komplexe betriebliche Entgeltsysteme oder Tarifverträge, deren Wirkungsmechanismen einzelne Benachteiligte nur schwer durchschauen und vor die Gerichte bringen können. Im Bereich der Entgeltgleichheit hat es zwar mehr Klagen gegeben. Eine Diskriminierung ist auch hier jedoch von der einzelnen Klägerin zumeist nur schwer zu beweisen, weil sie keinen Einblick in das gesamte Entlohnungssystem des Unternehmens oder Tarifvertrages und seine Hintergründe hat. Es fehlt im AGG an Untersuchungsrechten durch staatliche Stellen oder zumindest Auskunftsansprüche der Beschäftigten bezüglich der Entgeltstruktur. Nicht einmal die Antidiskriminierungsstelle des Bundes kann heute nach dem AGG Auskünfte von Unternehmen zur Entgeltstruktur verlangen. Sie darf „um Stellungnahme ersuchen “ ( $\$ 28$ Abs. 1 AGG), zur Antwort sind aber nur öffentliche Stellen im Bereich des Bundes verpflichtet ( $\mathbb{2} 28$ Abs. 2 AGG). Am konsequentesten wäre es, wenn im AGG die Beweislast generell umgekehrt würde, was die EU-Antidiskriminierungsrichtlinien allerdings nicht verlangen. In Österreich ist derzeit von der Frauenministerin vorgeschlagen worden, gesetzlich vorzuschreiben, dass alle Unternehmen ihre Entgeltsysteme offenlegen müssen, und bei nachgewiesener Entgeltdiskriminierung harte Bußgelder einzuführen.

Auch dort, wo solche Klagen gewonnen werden, erstreckt sich die Rechtskraftbindung nach dem AGG nur auf den Einzelfall. Die Tarifverträge verändern sich nur langsam, auch wenn die Gerichte in Einzelfällen einzelne Regelungen wegen Geschlechtsdiskriminierung für nicht anwendbar erklären. So hat das BAG Lohnabschlagsklauseln für Frauen schon 1955 für geschlechtsdiskriminierend erklärt. Die letzte tarifvertragliche Abschlagsklausel fiel aber erst 1972, also nach 17 Jahren!

Die Einzelklage Diskriminierter, wenn sie denn überhaupt gelingt, kann eben keine Veränderung der innerbetrieblichen

\footnotetext{
5 Vgl.z.B. 3. Bilanz Chancengleichheit: Europa im Blick, Berlin, April 2008.

6 Vgl. iwd Nr. 50 v. 11.12.2008 S. 8.

7 Einzelheiten siehe Raasch, Sibylle/Rastetter, Daniela: Die Umsetzung des AGG in den Betrieben, in: djbZ 1/2009 S. $21 \mathrm{ff}$.
} 
Entlohnungssysteme oder der Tarifverträge erzwingen. Es bedarf der Verbandsklage sowie direkter institutioneller Kontrollmöglichkeiten, um gegen Entgeltdiskriminierung in Kollektivsystemen wirksam vorgehen zu können.

\section{Verbandsklagerecht ins AGG}

Die Einführung eines Verbandsklagerechts für Antidiskriminierungsverbände gegen ganze Entlohnungssysteme und Tarifvertragsstrukturen würde den Druck in Richtung auf diskriminierungsfreie Entgeltsysteme im Vergleich zur bisherigen bloßen Einzelklage verstärken und wirksamer und schneller Abhilfe bei Entgeltdiskriminierung schaffen. Das Verbandsklagerecht sollte mit entsprechenden Auskunftsansprüchen der Verbände gegenüber den Tarifvertragsparteien und/oder den Unternehmen verbunden werden. Einzelne Klägerinnen würden dann nicht mehr durch Klagen belastet und eventuell ihr eigenes Beschäftigungsverhältnis und seine Weiterentwicklung gefährden müssen. Eine Breitenwirkung der Gerichtsentscheidung wäre gegeben. Insofern sollte das AGG hier über die EU-Antidiskriminierungsrichtlinien hinausgehen, die nur allgemein eine „Beteiligung“ der Verbände fordern.

\section{Kollektivverträge gesetzlich stärker kontrollieren}

Die Tarifvertragsparteien sind schon heute EU-rechtlich, verfassungsrechtlich und nach einfachem Gesetz - hier insbesondere $\int \mathbb{S} 2$ Abs. 1 Nr. 2, 7 Abs. 1 AGG - verpflichtet, ihre Tarifverträge diskriminierungsfrei zu gestalten. Adressat der Pflichten und Sanktionen ist allerdings nach $\mathbb{S} \mathbb{S} 11 \mathrm{ff}$. und $14 \mathrm{ff}$. AGG allein der Arbeitgeber. Die Kollektivorgane trifft lediglich eine allgemeine soziale Verantwortung, die in $\mathbb{1} 17$ Abs. 1 AGG nicht näher spezifiziert wird. Diskriminierende Bestimmungen in Tarifverträgen sind allerdings nach $\mathbb{} 7$ Abs. 2 AGG unwirksam. Diese Regelungen sind unstimmig und realitätsfern. Zwischen bloßer Verantwortung und sofortiger Unwirksamkeit klafft eine Lücke, welche durch die bereits kritisierte Haftungsreduktion zugunsten der einzelnen Arbeitgeber/innen aus $\mathbb{} 15$ Abs. 3 AGG nur unzureichend übertüncht wird.

Zwar steht den arbeitsrechtlichen Koalitionen die Tarifautonomie des Art. 9 Abs. 3 GG zur Seite. Diese entbindet aber nicht von den Diskriminierungsverboten bzw. von der Verpflichtung, ihren Entgeltsystemen diskriminierungsfreie Arbeitsbewertungssysteme zugrunde zu legen. Der Staat kann sich nicht mit dem Verweis auf die Tarifautonomie seiner eigenen Verpflichtung zum Schutz der Frauen vor Diskriminierung nach Gemeinschaftsrecht und dem Grundgesetz entziehen. Er ist nach Art. 3 Abs. 2 Satz 2 GG inzwischen sogar verfassungsrechtlich explizit verpflichtet, bestehende Benachteiligungen von Frauen zu beseitigen.

Seiner Schutzpflicht kann der Staat, ohne Art. 9 Abs. 3 GG zu tangieren, durch eine verfahrensmäßige Absicherung des Verbotes, wegen des Geschlechts zu diskriminieren, nachkommen. $\mathrm{Zu}$ unterscheiden wäre dabei zwischen künftig neu abzuschließenden und bereits bestehenden Tarifverträgen. Bloße Einsicht in die Ungerechtigkeit diskriminierender Entgeltsysteme reicht als Veränderungsmotor jedenfalls nicht aus. Eine
Veränderung ließe sich innerhalb des bisherigen Aushandlungssystems zumindest etwas leichter erreichen, wenn in den Verhandlungen um Entgeltregelungen die bisher benachteiligten Gruppen stärker repräsentiert wären als bisher, also Frauenquoten eingeführt würden.

Bei neuen Tarifverträgen könnte das Tarifvertragsgesetz dahingehend ergänzt werden, dass die Tarifvertragsparteien verpflichtet werden, ihren Entgeltsystemen diskriminierungsfreie Arbeitsbewertungssysteme zugrunde zu legen, jeden Tarifvertrag einem Diskriminierungscheck zu unterziehen und seine Diskriminierungsfreiheit zu bescheinigen, bevor er in Kraft treten kann. Dieser Check müsste einer staatlichen Stelle - in Betracht käme eine geschlechterparitätisch besetzte unabhängige Kommission, angebunden an die Antidiskriminierungsstelle des Bundes (ADS) - mit entsprechenden Unterlagen mitgeteilt und von ihr registriert werden, wonach der Tarifvertrag in Kraft treten kann. Bei Unklarheiten und Zweifeln sollten vor der Registrierung Nachfragen der Kommission zulässig sein. Antidiskriminierungsverbände sollten durch das AGG ermächtigt werden, in diese Unterlagen bei der Registrierungsstelle jederzeit Einsicht nehmen zu können und - für den Fall, dass sie einen Tarifvertrag im Hinblick auf ein Merkmal des $\mathbb{S}$ 1 AGG für diskriminierend halten - Verbandsklage zu erheben.

Schon bestehende Tarifverträge sollten innerhalb gesetzlich festzulegender Fristen nachträglich einer Überprüfung auf Diskriminierung durch die daran beteiligten Tarifvertragsparteien unterzogen und ggf. verändert werden müssen. Überprüfung, Ergebnis und Änderungen wären der Registrierungsstelle ebenso mitzuteilen wie im Fall neu abgeschlossener Tarifverträge. Kommt es bei der Überprüfung bestehender Tarifverträge nicht zu einer Einigung der Tarifvertragsparteien, könnte dem Tarifvertrag jedoch nicht einfach die weitere Wirksamkeit versagt werden. Denn sonst könnte eine Partei einen ihr unliebsamen Tarifvertrag auf diese Weise durch bloße Verweigerung des Konsenses beseitigen. Es wäre vielmehr eine Art Einigungsstelle im Sinne einer Entgeltgleichheitskommission unter einem unabhängigen Vorsitz einzusetzen, die über das weitere Vorgehen entscheidet. Auf die Person für den Vorsitz müssten sich beide Seiten einigen, anderenfalls bestellt das Arbeitsgericht den Vorsitz. ${ }^{8}$ Nur bei der Anwendung eines Tarifvertrages, der dieses Verfahren erfolgreich durchlaufen hat, dürfte die Haftung der Unternehmen auf Fälle grober Fahrlässigkeit beschränkt werden.

Für betriebliche Entgeltsysteme könnten entsprechende Regelungen auf der Ebene des Betriebsverfassungsgesetzes eingeführt werden, wobei notfalls eine betriebliche Einigungsstelle zu entscheiden hätte.

\section{Neue Arbeitsbewertungsmaßnahmen und -systeme}

Die Wissenschaft ist sich darüber einig, dass Verfahren zur analytischen Arbeitsplatzbewertung tendenziell besser zur dis-

8 Ähnlicher Vorschlag schon von Pfarr, Heide: Entgeltgleichheit in kollektiven Entgeltsystemen, in: Festschrift 50 Jahre BAG, München 2004 S. $779 \mathrm{ff}$. 
kriminierungsfreien Arbeitsbewertung geeignet sind als summarische. Denn erstere legen zuerst nach verschiedenen Merkmalen differenzierte, abstrakte Bewertungsgesichtspunkte fest und subsumieren dann jede einzelne konkrete Tätigkeit unter diese Merkmale. Erst die Gesamtsicht verschiedener Tätigkeiten und Tätigkeitsbestandteile auf einer beruflichen Position ergibt dann die Vergütung. Summarische Verfahren hingegen bilden nur grobe Fallgruppen, denen dann bestimmte Beschäftigtengruppen zugeordnet werden. Die Zuordnung erfolgt hier pauschal und weniger transparent. ${ }^{9}$

Die bisher verbreiteten Arbeitsbewertungsverfahren leiden jedoch alle daran, dass die Anforderungen an und der Einsatz von Fähigkeiten, wie sie häufiger auf typischen Frauenarbeitsplätzen gefordert und eher von Frauen erbracht werden, nicht als Qualifikation, Leistung oder Belastung gesehen und bewertet werden. Neben Geschicklichkeit, feinmotorischen Belastungen oder monotonen Arbeitsbedingungen im Produktionsbereich geht es hier vor allem um soziokommunikative Fähigkeiten und die sorgende Verantwortung für Menschen im Dienstleistungsbereich. Das Schweizer Verfahren zur Arbeitsplatzbewertung Abakaba ${ }^{10}$ bezieht auch solche Fähigkeiten, Anforderungen und Belastungen anteilig mit in die Bewertung ein, die eher von Frauen und auf typischen Frauenarbeitsplätzen zu erbringen sind. Dieses Verfahren wurde mit Unterstützung des Schweizer Staates entwickelt und ist in der Schweiz auch bereits mehrfach erfolgreich eingesetzt worden, vor allem von öffentlichen Einrichtungen. Abakaba ist jedoch nicht der einzige Neuansatz. Für Deutschland hat Karin Tondorf mit Logib-D ${ }^{11}$ inzwischen ebenfalls ein neues Instrument entwickelt.

Zur Unterstützung der Tarifvertragsparteien könnte bei der ADS oder einer anderen Registrierungsstelle ein Referat eingerichtet werden, das Informationen über besonders geschlechtergerechte Verfahren zur Arbeits- und Leistungsbewertung sammelt und Interessierten zur Verfügung stellt sowie Personalverantwortliche, Betriebs- und Personalräte, Tarifvertragsparteien, Gleichstellungsbeauftragte usw. berät.

Kein Arbeitsbewertungssystem verändert jedoch die Lohnsumme insgesamt, die in einem Betrieb oder Unternehmen zu verteilen ist. Ein Wechsel des Arbeitsbewertungssystems führt daher zwangsweise zu Umverteilungen innerhalb seines Anwendungsbereichs. Der Abbau von Diskriminierung muss letztlich von denjenigen „bezahlt“ werden, die bisher von dieser Benachteiligung profitiert haben. Von den bestehenden Entgeltsystemen profitieren naturgemäß vor allem diejenigen, die in den diesbezüglichen Verhandlungen am durchsetzungsstärksten waren, weil sie am besten personell repräsentiert wurden oder wegen ihrer Arbeitsmarktposition und ihrer Kampfbereitschaft am meisten Berücksichtigung erzwingen konnten - in der Praxis also Männer mit gewerkschaftlicher Organisierung und ohne Migrationshintergrund. Gegen den Widerstand dieser Akteure lassen sich neue Systeme mit Umverteilungseffekt nur schwer durchsetzen.

Gewisse Entspannung zwischen bisher benachteiligten Frauen und bisher begünstigten Männern könnte eine vorü- bergehende Besitzstandswahrung zugunsten der bislang Begünstigten sein, die sich erst schrittweise abbaut, zum Beispiel durch Anrechnung auf künftige Tariferhöhungen. Eine solche Besitzstandswahrung hätte aber die Unternehmensseite zu finanzieren. Insofern bietet sich eine mächtige Koalition zwischen den bislang begünstigten Männern und der Unternehmensseite zur Verhinderung grundlegender Veränderungen in Richtung auf weniger Diskriminierung der Frauen geradezu an. Diese Koalition ließe sich vielleicht dadurch aufbrechen, dass die Erhöhung der Frauenentgelte nur schrittweise über sogenannte Pay Equity Plans erfolgt. ${ }^{12}$ Allerdings verstieße diese weiche Lösung erneut gegen den gemeinschaftsrechtlichen Grundsatz der Entgeltgleichheit, wie er sich insbesondere aus Art. 141 EG als unmittelbar in Deutschland geltendem Recht ergibt, sodass eine solche nur schrittweise Anpassung des Entgelts von Männern und Frauen auch gewisse Rechtsprobleme aufwirft.

9 Vgl. Krell, Getraude/Winter, Regine: Anforderungsabhängige Entgeltdifferenzierung... in: Krell, Gertraude (Hrsg.): Chancengleichheit durch Personalpolitik, 5. Aufl. Wiesbaden 2008 S. $263 \mathrm{ff}$.

10 Näheres Katz, Christian P./Baitsch, Christof: Arbeit bewerten - Personal beurteilen, Zürich 2006.

11 Tondorf, Karin: „Logib-D“ - ein Weg zur Entgeltgleichheit, S. 130 in diesem Heft.

12 Vgl. Kocher, Eva: Individualansprüche als Bremse proaktiver Politiken der Entgeltgleichheit? In: Arioli, Katrin u.a. (Hrsg.): Wandel der Geschlechterverhältnisse durch Recht? Zürich, St. Gallen 2008, S. $135 \mathrm{ff}$. 